
Programa de Pós-Graduação em Engenharia de Produção - PPGEP
Laboratório de Qualidade de Vida - LaQVida
Universidade Tecnológica Federal do Paraná - UTFPR
Ponta Grossa - PR - Brasil
v. 04, n. 02, jul./dez. 2012, p. 25-37
DOI: $10.3895 / \mathrm{S} 2175-08582012000200003$

\author{
REVISTA BRASILEIRA DE \\ QUALIDADE DE VIDA
}

\title{
Atividade física e qualidade de vida em adultos mais velhos em Copacabana
}

\author{
Physical activity and quality of life in older adults in Copacabana
}

\begin{abstract}
Lilian Cristina Xavier Martins
Escola de Educação Física do Exército - EsEFEx - Rio de Janeiro - Brasil

lilitina@gmail.com

Leticia Fortes Legay

Instituto de Estudos em Saúde Coletiva, Universidade Federal do Rio de Janeiro, Departamento de Epidemiologia - Rio de Janeiro - Brasil

legay@iesc.ufr.j.br

Antonio Carlos Monteiro Ponce de Leon Instituto de Medicina Social, Universidade do Estado do Rio de Janeiro, Departamento de Epidemiologia - Rio de Janeiro - Brasil ponce@ims.uerj.br
\end{abstract}

\section{Resumo}

Pouco se sabe ainda no Brasil sobre a relação entre os níveis de atividade física (AF) e qualidade de vida $(\mathrm{QV})$ em pessoas aparentemente saudáveis. O objetivo do presente estudo foi investigar, nesse tipo de população, os fatores que influenciam a percepção da $\mathrm{QV}$, tendo como foco principal a prática de AF. Trata-se de um estudo transversal cuja amostra $(n=76)$ foi constituída de praticantes de natação ou hidroginástica e de conhecidos que não participavam destas atividades (método snow ball - bola de neve), todos moradores de Copacabana (abrangendo os distritos de: Copacabana e Leme). AF foi avaliada pelo Questionário de Baecke e QV pelo WHOQOL-Bref. As associações foram estimadas por meio de regressão linear múltipla (modelos lineares generalizados). A AF no lazer e em locomoção apresentou-se positivamente associado com todos os domínios da QV: físico $(\mathrm{p}=0,02)$, psicológico $(\mathrm{p}=0,02)$, social $(\mathrm{p}=0,03)$ e ambiental $(\mathrm{p}=0,02)$. Estes achados sugerem que AF pode beneficiar não só os domínios físicos (físico e ambiental), mas também nos domínios psicológico (psicológico e social) que compõem a avaliação da QV em populações semelhantes.

Palavras-chave: qualidade de vida, atividade física, doenças crônicas, saúde pública, envelhecimento.

\footnotetext{
Abstract

Little is known yet in Brazil about the relationship between levels of PA and quality of life (QOL) in apparently healthy people in Copacabana. The aim of this study was to investigate, in this population, the factors that influence the perception of QOL, focusing mainly on the practice of PA. This was a cross-sectional study and the sample consisted of 76 inhabitants of Copacabana (covering two districts: Copacabana and Leme) who used to practice swimming or hydro gymnastics and acquaintances who do not participate in those activities (snowball method). PA was
} 
assessed by the Baecke's Questionnaire and QOL by WHOQOL-Bref. Generalized Linear Models performed the analyses of linear regression. Leisure and locomotion PA was positively associated with all domains of QOL: physical $(\mathrm{p}=0.02)$, psychological $(\mathrm{p}=0.02)$, social $(\mathrm{p}=0.03)$ and environmental $(\mathrm{p}=0.02)$. These findings suggest that PA can benefit not only the physical domains (physical and environmental), but also the psychological domains (psychological and social) that comprise the assessment of QOL.

Keywords: quality of life, physical activity, chronic disease, public health, aging

\section{Introdução}

O conjunto de comportamentos que podem afetar a saúde do indivíduo está relacionado com o estilo de vida e inclui: tabagismo, consumo de álcool, hábitos alimentares, atividade física (AF), entre outros. A literatura apresenta amplas evidências dos benefícios da AF para o sistema cardiovascular e tratamento e prevenção da obesidade. Além disso, estudos mais recentes têm mostrado que a AF reduz a incidência de certos tipos de câncer (WARBURTON; NICOL; BREDIN, 2006), protege contra o declínio cognitivo (HENDRIE et al., 2006), degeneração macular relacionada à idade (KNUDTSON; KLEIN; KLEIN, 2006) e saúde mental (WARBURTON; NICOL; BREDIN, 2006). Outros fatores que estão relacionados com o estado de saúde são integrados ao contexto de vida: condições de pobreza, emprego, nutrição, entre outros. No entanto, apenas a partir da década de 80 tiveram início as discussões sobre a importância e o impacto das condições socioeconômicas, políticas e culturais sobre a saúde. Nesse contexto, configurou-se a noção de que a QV engloba diferentes dimensões e buscou-se uma melhor compreensão do fenômeno (SEIDL; ZANNON, 2004). Sob uma perspectiva de promoção da saúde, QV refere-se a uma condição de saúde que transcende a ausência de doença, envolvendo a noção de bem-estar. $\mathrm{O}$ conceito de QV da Organização Mundial da Saúde (OMS) (WHO, 1996) definiu QV como a percepção do indivíduo sobre sua posição na vida, no contexto da cultura e sistemas de valores, nos quais vive, e em relação aos seus objetivos, expectativas, padrões e preocupações (THE WHOQOL GROUP, 1995).

De acordo com Patrick e Ericksson (1993), QV refere-se à satisfação do indivíduo em relação às suas habilidades. Os autores exemplificam que uma pessoa com uma doença crônica ou deficiência pode ter uma alta QV, evitando depressão ainda que dependa de assistência pessoal, ao passo que uma pessoa sem doença e sem deficiência pode ter uma QV inferior, estar deprimido e infeliz com sua situação. Em adição, a QV relacionada à saúde é de grande importância em intervenções médicas devido às propriedades preditivas sobre prognósticos, estando associada à saúde mental (BHATTACHARYYA et al., 2007; OMS/OPAS, 2001; WEIDENHAMMER et al., 2006).

Nos processos de trabalho da sociedade moderna, as rotinas são cada vez mais automatizadas, consequentemente, um número crescente de pessoas tem cada vez menos oportunidade de praticar AF. Um estilo de vida sedentário está associado a risco aumentado para várias doenças crônicas, transtornos mentais e morte prematura (HALLAL et al., 2005). Além disso, a prática da AF está associada a uma menor taxa de mortalidade por todas as causas (SCHNOHR; SCHARLING; JENSEN, 2003).

Para uma maior compreensão dos fenômenos investigados é importante distinguir os conceitos de AF e exercício físico. AF é qualquer movimento corporal produzido pela contração do músculo esquelético que aumenta o gasto energético acima de um nível basal (US, 2009). Exercício físico é uma subcategoria de AF que é planejada, estruturada, realizado com movimentos repetitivos e intencionais visando objetivos de melhoria e/ou manutenção de um ou mais componentes da aptidão física (CASPERSEN; POWELL; CHRISTENSON, 1985).

A AF tem contribuído para melhorar a QV de diferentes tipos de pacientes, como pacientes com doenças cardiovasculares e com artrite, no último caso, aliviando o sofrimento, porque o 
exercício físico em pacientes com artrite diminui a dor com a qual têm que conviver (MEENAN; CALLAHAN; HELMICK, 1999). Além disso, estudos transversais, realizados na população em geral, têm mostrado consistentemente associação positiva entre $\mathrm{AF}$ e $\mathrm{QV}$ relacionada à saúde (BIZE; JOHNSON; PLOTNIKOFF, 2007; LIMA et al., 2009; SILVA et al., 2010; WENDEL-VOS et al., 2004). Em idosos, a AF está associada a mais anos de vida saudável no que diz respeito à saúde cardiovascular (HIRSCH et al., 2010).

Como em outros muitos países, no Brasil, a população está envelhecendo e é preciso atentar para o desenvolvimento de doenças crônicas e também para a QV desta população. O objetivo deste estudo foi investigar os fatores que influenciam a percepção da QV em adultos mais velhos, aparentemente saudáveis, tendo como foco principal a prática de AF.

\section{Métodos}

\subsection{Desenho e população de estudo}

Este é um estudo de caso, delineamento transversal, realizado no Rio de Janeiro em 2006, para estimar a relação entre $\mathrm{AF}$ e $\mathrm{QV}$ em pessoas adultas.

O procedimento de amostragem adotado é conhecido como snow ball (bola de neve). A amostra é obtida por meio de indicação de conhecidos dos sujeitos já participantes que possuem determinadas características de interesse (BIERNACKI; WALDORF, 1981). É uma técnica que tem sido utilizada para alcançar populações de difícil acesso, e é útil quando uma das metas é a redução de custos. Inicialmente, todos os participantes das atividades aquáticas (natação e hidroginástica) oferecidas para a comunidade do distrito de Copacabana, que engloba os bairros de Copacabana e Leme, em uma unidade militar, foram convidados a participar do estudo, e foram estimulados a trazer um conhecido que morasse nas proximidades. O critério de inclusão foi morar no distrito de Copacabana e o critério de exclusão foi ser menor de 18 anos.

Para o presente estudo considerou-se adultos mais velhos, pessoas acima de 50 anos.

Dos 120 sujeitos inscritos em hidroginástica e/ou natação, 43 foram excluídos pelo critério de exclusão. Dos 77 indivíduos elegíveis, 19 recusaram-se a participar. Assim, 58 foram convidados a preencher os questionários e convidar um conhecido que morava no bairro. Os participantes trouxeram 25 pessoas para o estudo, perfazendo um total de 83 participantes. A coleta de coleta de dados foi realizada entre janeiro e março de 2006.

O consentimento informado foi obtido de cada um dos participantes e os aspectos éticos deste estudo foram avaliados e aprovados pelo Comitê de Ética em Pesquisa do Instituto de Estudos em Saúde Coletiva da Universidade Federal do Rio de Janeiro, sendo que todos os procedimentos realizados estavam de acordo com os padrões éticos estabelecidos na Declaração de Helsinque 1964 e com a Resolução no 196, do Ministério da Saúde, de 10 de outubro de 1996.

\subsection{Variáveis}

\subsubsection{Qualidade de vida}

A QV, variável desfecho, foi estimada através do WHOQOL-Bref (WHO, 1996). O instrumento é validado para português do Brasil, sendo composto de 26 perguntas, constituindo-se num recordatório a respeito das duas últimas semanas, avaliando sob a ótica do sujeito suas percepções sobre sua QV nos domínios físico, psicológico, social e ambiental (FLECK et al., 2000). Os resultados são apresentados na forma de escores. 


\subsubsection{Atividade física}

O nível de AF, principal fator explicativo, foi estimado pelo Questionário de Baecke (BAECKE; BUREMA; FRIJTERS, 1982). Traduzido, adaptado e validado para português do Brasil (FLORINDO et al., 2004), o instrumento consiste em 16 perguntas que avaliam a AF, em um recordatório a respeito dos últimos 12 meses, em 3 dimensões: AF ocupacional (AFO), AF nos esportes/exercícios físicos no lazer (EFL) e AF de lazer e locomoção (ALL) apresentando resultados sob a forma de escore.

\subsubsection{Covariáveis}

As características socioeconômicas e demográficas (sexo, idade, renda, escolaridade, estado conjugal, aposentadoria, ter seguro saúde) autopercepção de saúde e informações sobre estilo de vida (fumo atual, consumo de qualquer quantidade de álcool nas atuas últimas semanas, buscar condicionamento físico e buscar socialização) foram as covariáveis selecionadas para integrar as análises.

\section{Análise estatística}

Inicialmente foi realizada a análise exploratória, sendo que para algumas análises gráficas, os escores (QV e AF) foram categorizados segundo tercis e classificados em níveis: baixo, intermediário e alto. $\mathrm{Na}$ análise bivariada foi aplicado o teste t de Student para amostras independentes, análise de variância (ANOVA One Way), regressão linear simples e os testes de correlação de Pearson e de Spearman.

Foram estabelecidas categorias de referência para as covariáveis: sexo (feminino), escolaridade (ensino superior), renda (mais de 20 salários mínimos), busca da melhoria no condicionamento físico (MCF) (sim) e possuir seguro de saúde (TSS) (sim) para a análise de regressão linear múltipla, realizada através do método Modelos Lineares Generalizados, e com base no procedimento stepwise que se utiliza o critério de informação de Akaike (AIC), a fim de selecionar covariáveis que seriam retiradas do modelo. A modelagem é implementada através da remoção de variáveis, uma de cada vez, até que o conjunto das variáveis que compõem o modelo final atinja o critério desejado de parcimônia. O modelo final é indicado quando a remoção de variáveis não causa diminuição no AIC. Os resíduos padronizados, relacionados com o modelo final, foram analisados quanto à normalidade para avaliar o ajuste geral dos modelos e, se necessário, procedeu-se à intervenção dos pesquisadores. Para se alcançar a adequação dos modelos, quando necessário, procedeu-se à transformação de algumas variáveis seguindo a indicação do método Box-Cox. Para todas as análises o nível de confiança foi de $95 \%$. Os dados foram digitados em MsAccess e analisados na Plataforma R.

\section{Resultados}

Depois de digitados os dados dos 83 participantes, 7 (8,43\%) foram excluídos por não terem preenchido o WHOQOL-Bref da forma mínima exigida. O número final de participantes foi de 76. Destes, $73,70 \%$ eram mulheres e a média de idade foi de 61 anos $( \pm 14,37)$, sendo que a maioria $(86,80 \%)$ estava acima dos 50 anos de idade. A maioria também era de casados $(56,58 \%)$, tinha 
ensino superior $(69,74 \%)$, apresentavam renda familiar entre cinco e dez salários mínimos brasileiros $(59,22 \%)$ e eram aposentados $(57,90 \%)$, como se pode obervar na Tabela 1 :

Tabela 1 - Características socioeconômicas e demográficas da população estudo

\begin{tabular}{|c|c|c|c|c|c|}
\hline Característica & Freq. & $\%$ & NA & $\%$ & \\
\hline \multicolumn{6}{|l|}{ Sexo } \\
\hline Homens & 20 & 26,30 & & & \\
\hline Mulher & 56 & 73,70 & & & \\
\hline Estado civil & & & 3 & 3,90 & \\
\hline Casado & 43 & 56,60 & & & \\
\hline Divorciado & 12 & 15,80 & & & \\
\hline Único & 8 & 10,50 & & & \\
\hline Viúva & 10 & 13,20 & & & \\
\hline Não relatado & 3 & 3,90 & & & \\
\hline \multicolumn{6}{|l|}{ Aposentado } \\
\hline Sim & 32 & 42,10 & & & \\
\hline Não & 44 & 57,90 & & & \\
\hline Escolaridade $^{\mathrm{a}}$ & & & 3 & 3,90 & \\
\hline Ensino superior & 53 & 69,74 & & & \\
\hline Ensino médio & 17 & 22,37 & & & \\
\hline Ensino fundamental & 3 & 3,95 & & & \\
\hline Renda $^{\mathrm{ab}}$ & & & 9 & 11,80 & \\
\hline Mais de 20 & 10 & 13,16 & & & \\
\hline Sobre $10-20$ & 22 & 28,95 & & & \\
\hline Sobre 5-10 & 13 & 17,11 & & & \\
\hline Sobre 2-5 & 16 & 21,05 & & & \\
\hline Até 2 & 6 & 7,89 & & & \\
\hline Nenhuma renda & 9 & 11,84 & & & \\
\hline \multicolumn{6}{|c|}{ Possuir seguro saúde (TSS) } \\
\hline Sim & 62 & 81,60 & & & \\
\hline \multirow[t]{2}{*}{ Não } & 14 & 18,40 & & & \\
\hline & Média & Mediana & $\mathrm{Sd}$ & Min & Max \\
\hline Idade & 61,31 & 61,00 & 14,37 & 24,00 & 87,00 \\
\hline
\end{tabular}

A Tabela 2 apresenta as informações autorreferidas sobre saúde e estilo de vida. A maioria era não fumante e apresentava algum tipo de dor, sendo que 40,80\% relataram hipertensão arterial. Observa-se que a prevalência de algum tipo de problema na coluna vertebral atingia quase metade dos participantes. 
Tabela 2 - Informações sobre saúde e estilo de vida a $(\mathbf{N}=76)$

\begin{tabular}{|c|c|c|}
\hline Informação & Freq. & $\%$ \\
\hline \multicolumn{3}{|l|}{ Fumo atual } \\
\hline Sim & 6 & 7,90 \\
\hline Não & 59 & 77,60 \\
\hline Ex-fumante & 11 & 14,50 \\
\hline \multicolumn{3}{|c|}{ Buscar melhor condicionamento físico } \\
\hline Sim & 42 & 55,30 \\
\hline Não & 34 & 44,70 \\
\hline \multicolumn{3}{|l|}{ Buscar socialização } \\
\hline Sim & 7 & 9,21 \\
\hline Não & 69 & 90,79 \\
\hline \multicolumn{3}{|l|}{ Dor recorrente } \\
\hline Sim & 44 & 57,90 \\
\hline Não & 32 & 42,10 \\
\hline \multicolumn{3}{|c|}{ Alguma dificuldade de movimento } \\
\hline Sim & 14 & 18,40 \\
\hline Não & 62 & 81,60 \\
\hline \multicolumn{3}{|l|}{ Problemas de coluna } \\
\hline Sim & 36 & 47,40 \\
\hline Não & 40 & 52,60 \\
\hline \multicolumn{3}{|l|}{ Tratamento ósseo } \\
\hline Sim & 25 & 32,90 \\
\hline Não & 51 & 67,10 \\
\hline \multicolumn{3}{|l|}{ Hipertensão } \\
\hline Sim & 31 & 40,80 \\
\hline Não & 45 & 59,20 \\
\hline \multicolumn{3}{|l|}{ Diabetes } \\
\hline Sim & 31 & 40,80 \\
\hline Não & 45 & 59,20 \\
\hline
\end{tabular}

Estatísticas descritivas das informações socioeconômicas e demográficas da população de estudo $(\mathrm{n}=76), 2006$.

Quanto à atividade física, $72,40 \%$ praticavam atividades aquáticas (natação ou hidroginástica) e 9,20\% informaram ter a caminhada como AF primeiro no esporte/exercício. Outras categorias de AF somadas totalizaram 13,20\%. Entre os que tinham como primeiro esporte/exercício atividades aquáticas, também praticavam a caminhada (como segundo esporte/exercício) $(35,50 \%)$.

A Tabela 3 apresenta os resultados de QV e de AF. A média de QV foi de 108,88 $( \pm 14,54)$ e a mediana foi de 109,00 e $80,00 \%$ dos participantes exibiram 100 ou mais pontos em QV. A média de prática AF foi $8,61( \pm 1,85)$ e cada uma das dimensões apresentou mediana próxima da média, indicando que a distribuição seria normal ou aproximadamente normal.

Tabela 3 - Distribuição de atividade física e qualidade de vida (N=76)

\begin{tabular}{lrrrrr}
\hline & Média & Mediana & \multicolumn{1}{c}{ DP } & \multicolumn{1}{c}{ Min } & Max \\
\hline Qualidade de Vida (QV) & 108,88 & 109,00 & 14,54 & 64,00 & 133,00 \\
Domínio físico & 23,80 & 24,00 & 3,08 & 14,00 & 30,00 \\
Domínio psicológico & 21,04 & 21,00 & 3,04 & 12,00 & 27,00 \\
Domínio social & 11,13 & 11,00 & 2,11 & 4,00 & 15,00 \\
Domínio ambiental $^{a}$ & 52,91 & 53,00 & 8,30 & 29,00 & 69,00 \\
Atividade física (AF) & 8,61 & 9,00 & 1,85 & 1,75 & 11,50 \\
Ocupacional PA (AFO) & 2,43 & 2,75 & 0,93 & 0,00 & 3,50 \\
Esportes e exercícios no lazer (EFL) & 3,50 & 3,50 & 0,86 & 0,25 & 4,75 \\
De lazer e locomoção PA (ALL) & 2,68 & 2,75 & 0,69 & 1,00 & 4,75 \\
\hline
\end{tabular}

${ }^{\mathrm{a}}$ NAs (dados ausentes): 18 (23,70\%).

Nota: SLT + LLPA $=6,18$. 
A Figura 1 mostra a distribuição da QV por níveis de AF.

Figura 1 - Qualidade de vida segundo atividade física ${ }^{a}$

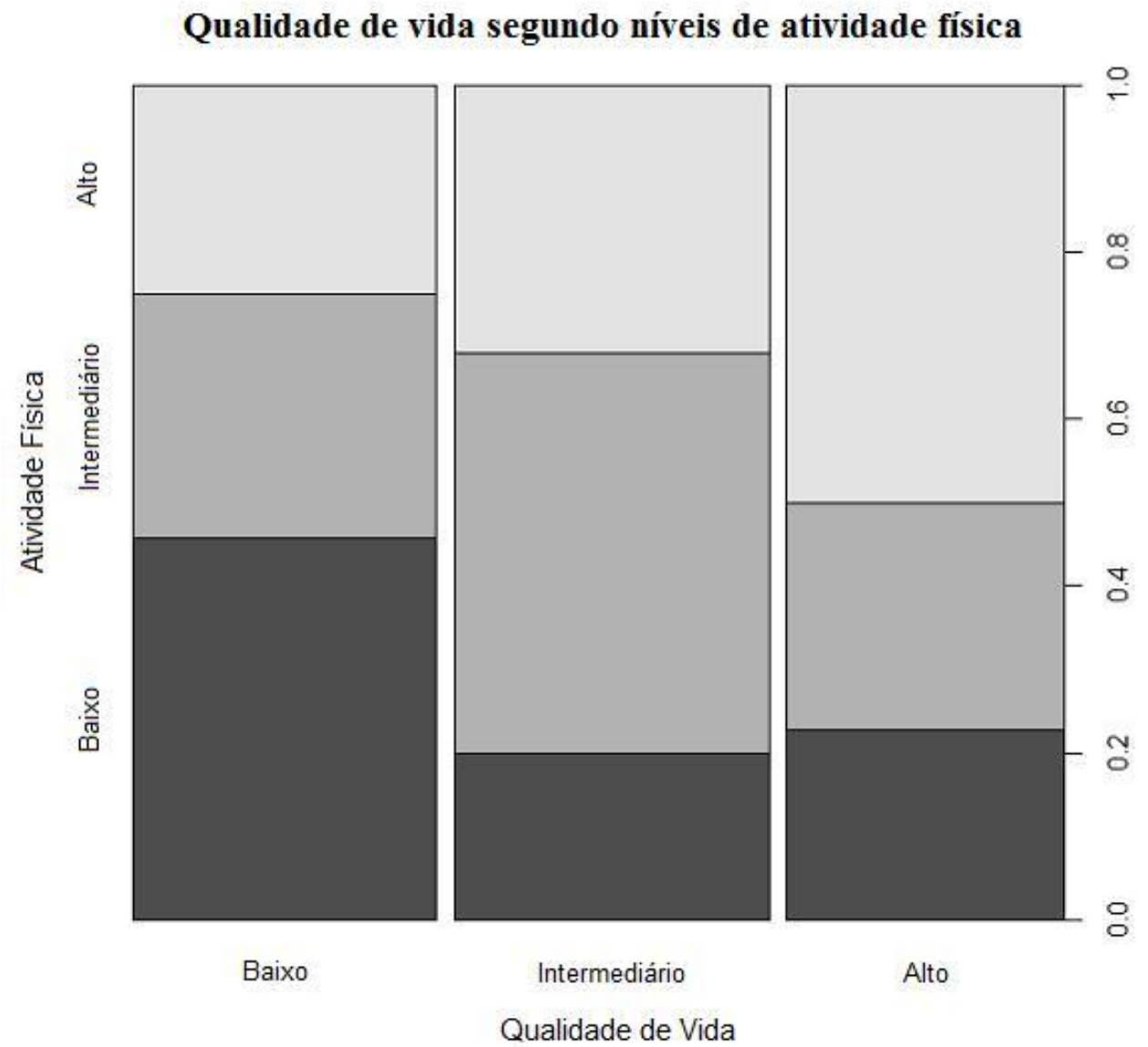

a Distribuição de qualidade de vida segundo atividade física na população de estudo ( $\mathrm{n}=$
76), 2006. O escore total de qualidade de vida e o escore total de atividade física foram
categorizados, segundo tercis, e classificados em níveis baixo, intermediário e alto.

$\mathrm{Na}$ análise bivariada as seguintes variáveis apresentaram-se positivamente associadas com QV: ter seguro de saúde (TSS) $(\mathrm{p}=0,01)$, renda $(\mathrm{p}<0,001)$, outras atividades físicas no lazer e em locomoção (ALL) $(\mathrm{p}<0,001)$ e praticar atividade física visando melhora no condicionamento físico $(\mathrm{MCF})(\mathrm{p}=0,02)$. Estas variáveis fizeram parte do modelo completo para a $\mathrm{QV}$, mais escolaridade, sexo e idade.

A análise bivariada foi realizada também em relação aos domínios que compõem a QV. Ao domínio físico somente ALL estava significativa e positivamente associada ( $\mathrm{p}<0,05)$. Ao domínio psicológico, escolaridade $(\mathrm{p}<0,001)$ e $\operatorname{ALL}(\mathrm{p}=0,01)$ associaram-se positivamente. Ao domínio social somente ALL $(\mathrm{p}=0,01)$ estava significativa e positivamente associada. Estavam positivamente associadas ao domínio ambiental: renda $(p=0,00)$ e ALL $(p=0,04)$. AFO estava negativamente associada $(\mathrm{p}=0,03)$. Todas as variáveis que mostraram associação significativa foram consideradas para os respectivos modelos sob a análise multivariada. Para todas as análises subsequentes, os modelos foram ajustados através da aplicação do procedimento stepwise.

$\mathrm{Na}$ modelagem estatística, para o escore total de QV, o modelo completo incluiu as seguintes variáveis: renda, MCF, TSS, AFO, ALL, SLT, sexo e idade. No modelo final, apenas 
ALL ( $\mathrm{p}=0,01)$ mostrou associação positiva. A análise dos resíduos não mostrou normalidade dos dados, portanto, a transformação Box-Cox foi aplicada. Como resultado, a QV foi elevada ao quadrado.

Após o procedimento stepwise permaneceram no modelo final as variáveis: renda, TSS e ALL. Independentemente de idade, sexo e escolaridade, essas variáveis permaneceram associadas à QV na população de estudo (Tabela 4).

Tabela 4 - Qualidade de vida por variáveis socioeconômicas, demográficas e de estilo de vida

\begin{tabular}{|c|c|c|c|}
\hline Qualidade de vida $^{a}$ & Coeficientes & IC $(95 \%)$ & $\boldsymbol{P}$ \\
\hline \multicolumn{4}{|l|}{ Renda } \\
\hline Sobre $20^{\mathrm{b}}$ & & & 0,00 \\
\hline Sobre $10-20$ & 697,20 & $(-1.109,74 ; 2.505,00)$ & 0,45 \\
\hline Sobre 5-10 & 491,20 & $(-1.524,17 ; 2.506,82)$ & 0,63 \\
\hline Sobre $2-5$ & $-2.457,40$ & $(-4.380,52 ;-534,31)$ & $0,02 *$ \\
\hline Até 2 & $-1.569,60$ & $(-4.212,25 ; 1.073,05)$ & 0,25 \\
\hline \multicolumn{4}{|l|}{ Ter seguro saúde (TSS) } \\
\hline $\mathrm{Sim}^{\mathrm{b}}$ & & & 0,00 \\
\hline Não & $-1.614,80$ & $(-9,02 ; 3.238,70)$ & 0,06 \\
\hline \multicolumn{4}{|l|}{ Atividade física } \\
\hline De lazer e locomoção (ALL) & $1.221,10$ & $(392,30 ; 2.049,95)$ & $0,01 *$ \\
\hline
\end{tabular}

A análise por domínios da QV apresenta-se na Tabela 5. No modelo final para o domínio físico permaneceram renda, TSS e ALL. Apenas ALL estava positivamente associado ao domínio físico apresentando um coeficiente $(\beta)$ de 1,21 e um intervalo de confiança (IC) a 95\% entre 0,24 e 2.17. No domínio psicológico, o modelo final foi composto por: escolaridade, sexo, idade e ALL. A este domínio, o nível médio de escolaridade estava negativamente associado ( $\beta=-1,66$ IC95\% -3,01; $-0,31, p=0,02)$ e ALL $(\beta=1,21$ IC95\% 0,$27 ; 2,14, p=0,02)$ estava positivamente associado. No domínio social, o modelo final foi composto por idade e ALL. Apenas ALL estava associada à QV ao domínio social e de forma positiva $(\beta=0,84$ IC95\% 0,$10 ; 1,60, p=0,03)$. No domínio ambiental, o modelo final foi composto por renda, AFO e ALL. Os dois níveis mais baixos de renda estavam negativamente associados a este domínio. ALL $(\beta=2,93$ IC95\% 0,55; 5,31, $p=0,02)$ estava positivamente associada. 
Tabela 5 - Qualidade de vida, modelos finais por domínios $(\mathrm{N}=76)$

\begin{tabular}{|c|c|c|c|}
\hline Domínio / Variáveis & $\square^{\mathbf{a}}$ & IC $(95 \%)$ & $\boldsymbol{P}$ \\
\hline \multicolumn{4}{|l|}{ Domínio físico } \\
\hline \multicolumn{4}{|l|}{ Renda } \\
\hline \multicolumn{4}{|l|}{ Mais de $20^{b}$} \\
\hline Sobre $10-20$ & 0,70 & $(-1,39 ; 2,80)$ & 0,51 \\
\hline Sobre $5-10$ & 1,27 & $(-1,07 ; 3,61)$ & 0,30 \\
\hline Sobre $2-5$ & $-1,42$ & $(-3,66 ; 0,81)$ & 0,22 \\
\hline Até 2 & 0,28 & $(-2,79 ; 3,34)$ & 0,86 \\
\hline \multicolumn{4}{|l|}{ Ter seguro saúde (TSS) } \\
\hline \multicolumn{4}{|l|}{$\operatorname{Sim}^{\mathrm{b}}$} \\
\hline Não & $-1,90$ & $(-3,78 ;-0,02)$ & 0,05 \\
\hline \multicolumn{4}{|l|}{ Atividade física } \\
\hline De lazer e locomoção (ALL) & 1,21 & $(0,24 ; 2,17)$ & $0,02 *$ \\
\hline \multicolumn{4}{|l|}{ Domínio psicológico } \\
\hline \multicolumn{4}{|l|}{ Escolaridade } \\
\hline \multicolumn{4}{|l|}{ Ensino superior ${ }^{b}$} \\
\hline Ensino médio & $-1,66$ & $(-3,01 ;-0,31)$ & $0,02 *$ \\
\hline Ensino fundamental & $-2,18$ & $(-4,62 ; 0,26)$ & 0,09 \\
\hline Idade & $-0,01$ & $(-0,05 ; 0,03)$ & 0,51 \\
\hline \multicolumn{4}{|l|}{ Gênero } \\
\hline \multicolumn{4}{|l|}{ Feminino ${ }^{b}$} \\
\hline Masculino & $-0,83$ & $(-2,10 ; 0,43)$ & 0,20 \\
\hline \multicolumn{4}{|l|}{ Atividade física } \\
\hline De lazer e locomoção (ALL) & 1,21 & $(0,27 ; 2.14)$ & $0,02 *$ \\
\hline \multicolumn{4}{|l|}{ Domínio social } \\
\hline Idade & $-0,01$ & $(-0,04 ; 0,02)$ & 0,60 \\
\hline \multicolumn{4}{|l|}{ Atividade física } \\
\hline De lazer e locomoção (ALL) & 0,84 & $(0,10 ; 1,60)$ & $0,03^{*}$ \\
\hline \multicolumn{4}{|l|}{ Domínio ambiental } \\
\hline \multicolumn{4}{|l|}{ Renda } \\
\hline \multicolumn{4}{|l|}{ Mais de $20^{b}$} \\
\hline Sobre $10-20$ & 1,55 & $(-3,58 ; 6,69)$ & 0,56 \\
\hline Sobre $5-10$ & 0,05 & $(-5,66 ; 5,78)$ & 0,98 \\
\hline Sobre 2-5 & $-8,00$ & $(-13,51-2,50)$ & $0,01 *$ \\
\hline Até 2 & $-8,25$ & $(-15,31 ;-1,19)$ & $0,03 *$ \\
\hline \multicolumn{4}{|l|}{ Atividade física } \\
\hline Atividade física ocupacional (AFO) & $-1,41$ & $(-3,32 ; 0,50)$ & 0,15 \\
\hline De lazer e locomoção PA (ALL) & 2,93 & $(0,55 ; 5,31)$ & $0,02 *$ \\
\hline
\end{tabular}

\footnotetext{
${ }^{\mathrm{a}}$ Coeficientes.

${ }^{\mathrm{b}}$ Referência categoria.

$* \mathrm{P}<0,05$.
}

\section{Discussão}

Os principais achados foram que, para os adultos mais velhos, as atividades físicas diferentes de esportes ou exercícios no lazer apresentaram-se positivamente associados à QV tanto com o escore total quanto com os domínios. Estas associações permaneceram mesmo após ajustar por idade, sexo, estado civil, aposentadoria, renda, estilo de vida e estado de saúde. Pessoas com altos escores em ALL também tiveram altos escores em QV. Isso mostra que a AF no tempo de lazer contribui para a QV em adultos mais velhos, aparentemente saudáveis. Tais resultados estão corroborados na literatura (ACREE et al., 2006; WENDEL-VOS et al., 2004).

Em relação aos domínios de QV, as atividades físicas no tempo de lazer e locomoção (ALL) estavam positivamente associadas ao domínio físico, o que é consistente com a literatura (WENDEL-VOS et al., 2004). 
O nível médio de escolaridade mostrou-se negativamente associado com o domínio psicológico, o que não aconteceu em pessoas com nível fundamental. Nível de escolaridade é um fator importante preditor para uma pior percepção de QV: pessoas com mais anos de escolaridade apresentam mais QV quando comparados àquelas com menos anos (LIMA et al., 2009). Os resultados mostraram que pessoas com mais ALL apresentaram mais QV no domínio psicológico (Tabela 4). Estes achados sugerem que a prática de atividades físicas no lazer, diferente de esportes ou exercícios formais, contribui para a QV também no domínio psicológico. A maioria dos estudos transversais não encontrou associação entre AF e os componentes psicológicos da QV (domínios psicológico e social) (WENDEL-VOS et al., 2004). Entretanto, os resultados do presente estudo concordam com os achados recentes de Silva et al. (2010), que também encontraram esta associação. Em estudos longitudinais esta associação é consistente com a literatura (WENDELVOS et al., 2004).

A associação inversa da QV com a idade é esperada. No presente estudo isto foi observado apenas no domínio social. Nos demais domínios este efeito não se apresentou.

No domínio social, ALL estava positivamente associada à QV. Em outras palavras, a prática de atividades físicas no tempo de lazer, diferente de esportes ou exercícios formais, contribui para a QV também no domínio social. Novamente, os resultados concordam com os achados de Silva et al. (2010). A prática de AF é um comportamento do estilo de vida, portanto, pode ser alterado para beneficiar a saúde. Nesse sentido, estudos têm relatado que o aumento do tempo dedicado a AF contribui para um funcionamento social mais satisfatório, independentemente da intensidade da atividade (WENDEL-VOS et al., 2004). Isto pode explicar a associação positiva da AF com o domínio social encontrada no presente estudo e no estudo de Silva et al. (2010).

Ao domínio ambiental, os dois níveis mais baixos de renda estavam negativamente associados, o que é consistente com a literatura (SILVA et al., 2010). ALL estava positivamente associado a este domínio. Este resultado pode ser explicado porque passar algum tempo de lazer em atividades ao ar livre, em ambientes favoráveis contribui para o aumento da prática de atividades físicas (FRANZINI et al., 2010; SAELENS et al., 2003; SILVA et al., 2010; WENDEL-VOS et al., 2004). A cidade do Rio de Janeiro oferece muitas possibilidades de atividades junto à natureza devido à sua rica paisagem natural. Em especial, a orla de suas praias representa um ambiente bastante favorável para a prática de diversos tipos de atividade física.

Embora diversos problemas de saúde tenham sido relatados pelos participantes (Tabela 2), nenhum destes mostrou qualquer associação com a QV. Observando os níveis de QV, AF e as informações de saúde, pode-se dizer que os problemas de saúde não afetaram a QV destas pessoas.

A população do estudo era homogênea quanto à escolaridade, portanto, não houve associação estatística com o escore total de QV, como seria esperado (SALAFFI et al., 2009). A maioria dos participantes das atividades aquáticas praticava também caminhada. Estudo conduzido em São Paulo apresentou resultados semelhantes em idosos de baixo nível socioeconômico (INÁCIO; SALVADOR; FLORINDO, 2011). Para a maioria dos indivíduos que participaram deste estudo, o escore de QV foi acima de 100 (80\%), exibindo os altos níveis de QV nesta população. Além disso, a maior parte dos participantes do estudo apresentava níveis de AF variando de intermediário a alto, que pode ser explicado pelo ambiente agradável e razoavelmente seguro, componentes que estimulam a prática de atividades físicas de lazer ao ar livre (TAYLOR; SALLIS; NEEDLE, 1985; ZENK et al., 2009) como é o caso de caminhar na calçada da praia e/ou praticar natação/hidroginástica.

Apesar de a amostra ter sido constituída, na maior parte, de pessoas mais velhas (média de

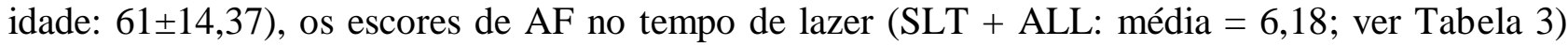
podem ser considerados altos níveis se comparados ao estudo conduzido em uma população de adultos jovens (média de idade: 32,6 $\pm 3,1$ ) em São Paulo que exibiu uma média nos escores de AF no lazer de 7,4 ( $\pm 0,1)$ (FLORINDO; LATORRE, 2003).

A literatura é abundante sobre os benefícios da AF para a saúde relacionados com a QV tanto em estudos longitudinais quanto em seccionais (WENDEL-VOS et al., 2004). Os resultados 
do presente estudo indicam que as atividades físicas no lazer, principalmente aqueles diferentes de esportes ou exercícios físicos formais (ginástica ou musculação), como, por exemplo, caminhar e/ou andar de bicicleta estão associados a mais a QV em todos os domínios (físico, social, psicológico e ambiental), como também ao escore total.

A maioria dos estudos transversais tem demonstrado a associação de AF com os componentes físicos da QV, enquanto estudos longitudinais, predominantemente, têm mostrado associações com seus componentes psicológicos (WENDEL-VOS et al., 2004). O presente estudo, embora de desenho transversal, encontrou associação da AF no lazer com todos os domínios da QV, tanto com aqueles de caráter físico (físico e ambiental) como para os domínios de caráter psicológico (psicológico e social), e estas associações permaneceram mesmo após ajustes para idade, sexo, nível educacional, renda, hábitos de vida e aspectos de saúde autorreferidos. Estes achados estão na mesma direção dos resultados de Lima et al. (2009).

No presente estudo as atividades físicas no lazer, diferentes de esportes formais ou exercícios físicos, estavam positivamente associadas com o escore global de QV, bem como com cada um dos domínios mostrando que, pelo menos para os adultos mais velhos, esse tipo de AF é importante para a QV. Outro achado importante foi que a presença de problemas de saúde não afetou o comportamento $\mathrm{AF}$ e nem a $\mathrm{QV}$ dos participantes. Estudos futuros poderiam investigar um possível efeito modificador da $\mathrm{AF}$ sobre a $\mathrm{QV}$ e agravos à saúde.

Dentre as limitações do estudo está que a amostra foi do tipo não probabilística, e neste caso, as inferências estão restritas a populações com características semelhantes às da população de estudo, como os habitantes de outros bairros (praias), no Rio de Janeiro, como Ipanema, Leblon e Barra da Tijuca.

O sedentarismo aumenta os riscos de várias doenças e a investigação sobre os níveis de AF e sua associação com a QV é uma questão importante, ficando ressaltada a relevância deste trabalho. Considerando que raros estudos no Brasil investigaram QV em pessoas aparentemente saudáveis, este estudo contribuiu para o conhecimento sobre a relação entre os níveis de AF e QV, pode-se sugerir que seja conduzido estudo de base populacional para confirmar estes achados.

A AF além de produzir benefícios de saúde também oferece benefícios para QV, por conseguinte, incentivar a prática de AF é uma importante estratégia de promoção da saúde, porque o custo é relativamente barato e, mesmo que o efeito possa ser relativamente pequeno, haveria um impacto dramático sobre gastos em saúde (HENDRIE et al., 2006).

Em conclusão, a natureza específica da população do estudo limita a extrapolação dos resultados apenas para populações semelhantes a esta e sugere-se que outros estudos sejam realizados no Brasil a respeito da relação entre prática de AF no lazer e a QV, em sua avaliação geral e por os domínios da QV em pessoas aparentemente saudáveis.

\section{Referências}

ACREE, L. S. et al. Physical activity is related to quality of life in older adults. Health Quality of Life Outcomes, v. 4, p. 37, 2006.

BAECKE, J. A.; BUREMA, J.; FRIJTERS, J. E. A short questionnaire for the measurement of habitual physical activity in epidemiological studies. Am. J. Clinical Nutrition, v. 36, p. 936-942, nov. 1982.

BHATTACHARYYA, M. R.; PERKINS-PORRAS, L.; WHITEHEAD, D. L.; STEPTOE, A. Psychological and clinical predictors of return to work after acute coronary syndrome. European Heart Journal, v. 28, n. 2, p. 160-165, jan. 2007. 
BIERNACKI, P.; WALDORF, D. Snowball sampling: problems and techniques of chain referral sampling. Sociological Methods and Research, v. 10 n. 2, p. 141-163, nov. 1981.

BIZE, R.; JOHNSON, J. A.; PLOTNIKOFF, R. C. Physical activity level and health-related quality of life in the general adult population: a systematic review. Preventive Medicine, v. 45, n. 6, p. 401-415, dez. 2007.

CASPERSEN, C. J.; POWELL, K. E.; CHRISTENSON, G. M. Physical activity, exercise, and physical fitness: definitions and distinctions for health-related research. Public Health Rep, v. 100, n. 2, p. 126-131, abr. 1985.

FLECK, M. P. et al. Aplicação da versão em português do instrumento abreviado de avaliação da qualidade de vida 'WHOQOL-bref'. Revista de Saúde Pública, v. 34, p. 178-183, 2000.

FLORINDO, A. A.; LATORRE, M. R. Validation and reliability of the Baecke questionnaire for the evaluation of habitual physical activity in adult men. Revista Brasileira de Medicina do Esporte, v. 9, p. 129-135, 2003.

FlORINDO, A. A.; LATORRE, M. R.; JAIME, P. C.; TANAKA, T.; ZERBINI, C. A. D. F. Metodologia para a avaliação da atividade física habitual em homens com 50 anos ou mais. Revista de Saúde Pública, v. 38, p. 307-314, 2004.

FRANZINI, L. et al. Neighborhood characteristics favorable to outdoor physical activity: disparities by socioeconomic and racial/ethnic composition. Health Place, v. 16, n. 2, p. 267-274, mar. 2010.

HALLAL, P. C. et al. Physical activity in adults from two Brazilian areas: similarities and differences. Cadernos de Saúde Pública, v. 21, n. 2, p. 573-580, abr. 2005.

HENDRIE, H. C. et al. The NIH Cognitive and Emotional Health Project. Report of the Critical Evaluation Study Committee. Alzheimer's \& dementia: the journal of the Alzheimer's Association, v. 2, n. 1, p. 12-32, jan. 2006.

HIRSCH, C. H. et al. Physical activity and years of healthy life in older adults: results from the cardiovascular health study. Journal of aging and physical activity, v. 18, n. 3, p. 313-334, jul. 2010 .

INÁCIO, R. F.; SALVADOR, E. P.; FLORINDO, A. A. Análise Descritiva da Prática de Atividade Física no Lazer de Idosos Residentes em Uma Região de Baixo Nível Socioeconômico da Zona Leste de São Paulo, SP. Revista Brasileira de Atividade Física e Saúde, v. 16, n. 2, p. 150-155, 2011.

KNUDTSON, M. D.; KLEIN, R.; KLEIN, B. E. K. Physical activity and the 15-year cumulative incidence of age-related macular degeneration: the Beaver Dam Eye Study. British Journal of Ophthalmology, v. 90, n. 12, p. 1461-1463, dez. 2006.

LIMA, M. G. et al. Health related quality of life among the elderly: a population-based study using SF-36 survey. Cadernos de Saúde Pública, v. 25, p. 2159-2167, 2009.

MEENAN, R. F., CALlAHAN, L. F. AND HELMICK, C. G. The National Arthritis Action Plan: A public health strategy for a looming epidemic. Arthritis \& Rheumatism, v. 12, p. 79-81, 1999. 
OMS/OPAS. Relatório sobre a saúde no mundo 2001 - Saúde Mental: Nova Concepção, Nova Esperança. OPAS, Washington. Organização Pan-Americana da Saúde/Organização Mundial da Saúde, Washington, p. 43-45, 2001.

PATRICK, D. L.; ERICKSSON, P. Quality of Life in Health Care Evaluation and Resource Allocation. New York: Oxford University Press, 1993.

SAELENS, B. E.; SALLIS, J. F.; BLACK, J. B.; CHEN, D. Neighborhood-based differences in physical activity: an environment scale evaluation. American Journal of Public Health, v. 93, n. 9, p. 1552-1558, set. 2003.

SALAFFI, F. et al. Health-related quality of life in fibromyalgia patients: a comparison with rheumatoid arthritis patients and the general population using the SF-36 health survey. Clinical and Experimental Rheumatology, v. 27, n. 5 Suppl 56, p. 567-574, out. 2009.

SCHNOHR, P.; SCHARLING, H.; JENSEN, J. S. Changes in leisure-time physical activity and risk of death: an observational study of 7,000 men and women. American Journal Epidemiology, v. 158 , n. 7, p. 639-644, 01 out. 2003.

SEIDL, E. M. F.; ZANNON, C. M. L. D. C. Qualidade de vida e saúde: aspectos conceituais e metodológicos. Cadernos de Saúde Pública, v. 20, p. 580-588, 2004.

SILVA, R. S.; SILVA, I. da; SILVA, R. A. da; SOUZA, L.; TOMASI, E. [Physical activity and quality of life]. Ciência e Saúde Coletiva, v. 15, n. 1, p. 115-120, jan. 2010.

TAYLOR, C. B.; SALLIS, J. F.; NEEDLE, R. The relation of physical activity and exercise to mental health. Public Health Reports, v. 100, n. 2, p. 195-202, abr. 1985.

THE WHOQOL GROUP. The World Health Organization Quality of Life assessment (WHOQOL): position paper from the World Health Organization. Social Science \& Medicine (1982), v. 41, n. 10, p. 1403-1409, nov. 1995.

U.S. DEPARTMENT OF HEALTH AND HUMAN SERVICES. Physical activity guidelines for Americans. The Oklahoma Nurse, v. 53, n. 4, p. 25, 2009.

WARBURTON, D. E.; NICOL, C. W.; BREDIN, S. S. Health benefits of physical activity: the evidence. Canadian Medical Association Journal, v. 174, n. 6, p. 801-809, 14 mar. 2006.

WEIDENHAMMER, W.; WESSEL, A.; HUTTER, A.; MELCHART, D.; SCHRODER, A. [Chronic fatigue in complementary rehabilitative medicine--predictors of the outcomes]. Rehabilitation (Stuttg), v. 45, n. 5, p. 299-308, out. 2006.

WENDEL-VOS, G. C.; SCHUIT, A. J.; TIJHUIS, M. A.; KROMHOUT, D. Leisure time physical activity and health-related quality of life: cross-sectional and longitudinal associations. Quality of Life Research, v. 13, n. 3, p. 667-677, abr. 2004.

WHO, W. H. O. What quality of life? The WHOQOL Group. World Health Organization Quality of Life Assessment. World Health Forum, v. 17, n. 4, p. 354-356, 1996.

ZENK, S. N. et al. Neighborhood environment and adherence to a walking intervention in African American women. Health Education \& Behavior, v. 36, n. 1, p. 167-181, fev. 2009. 